\title{
Analysis and Optimal Design of Induction Heating Cookers
}

\author{
Muhammad Humza* and Byungtaek Kim ${ }^{\dagger}$
}

\begin{abstract}
This paper deals with the optimal design of induction heating cooker starting from the analytical analysis of the cooker model. The analytical analysis method is based on the construction of the equivalent circuit of the cooker in which the circuit parameters are calculated from the geometries in a very simple way. By using the analysis method of equivalent circuit, the numerous combinations of primary winding coils for the specific rated power are obtained in fast computation time for a given secondary conductor. Through the calculation procedures, the optimal number of turns and their optimal positions can be obtained with which the cooker provides the highest efficiency at the rated power. The effectiveness and accuracy of the proposed analysis and design are verified through finite element analysis for practical and designed models.
\end{abstract}

Keywords: Analysis of induction cooker, Equivalent circuit, Optimal design of primary coil

\section{Introduction}

Traditional ways of cooking are hanging the pot on fire which is produced by burning of wood, coal or gas. These traditional ways have a lot of draw backs. The gas burner transfer only $35 \%$ to $40 \%$ heat to the pan, which means large portion of heat is wasted and these methods of cooking consumed a lot of time for cooking. The burning of gas also produces the $\mathrm{CO}_{2}$ and other hazardous products which are unfriendly to the environment. On the other hand, induction heating cooker is friendly to environment and offers a lot of benefits such as high heating efficiency, fast, safe, clean, and cheap cooking. Induction heating is significantly energy and time saving [1]. Induction heating cooker consists of basically a heating coil (primary winding) and vessel or pot (secondary winding). The heating coil is placed underneath a conductive cooking vessel with a thermal insulator between them. A supply of medium frequency is applied to the heating coil, usually by means of power converter. It generates magnetic field which induces the current in the vessel which leads to the desired heating of vessel for the cooking purpose.

In the past, most designers have used FEM for design of the induction cooker which is accurate but much time consuming process [2-6]. This study proposes the novel method of analysis and optimal design of induction heating cooker by constricting the equivalent circuit. The equivalent circuit is constructed by using the analytical analysis from the geometries of a model. To get the circuit parameters of the secondary winding, it is divided into small elements to avoid from the skin and proximity effects. The depth of each element is selected less than the skin depth at desired

$\dagger$ Corresponding Author: Dept. of Electrical Engineering, Kunsan National University, South Korea. (btkim@kunsan.ac.kr)

* Dept. of Electrical Engineering, Kunsan National University, South Korea. (engr.humza7@gmail.com)

Received: April 14, 2016; Accepted: May 16, 2016 frequency. The self and mutual inductances of these elements are calculated by using the classical formulas obtained from the Biot-Savart law. The concrete equivalent circuit is constructed with the obtained parameters for the both primary and secondary windings. By solving the complicated coupled voltage circuit, a simplified equivalent circuit is obtained. The equivalent circuit provides an easy way to check the performance characteristics of induction heating cooker. This proposed designed method provides an easy and optimized way to calculate the number of turns of primary winding as well as the best positions for the insertion of coils on the primary side for the rated power. In this method the number of turns and their positions can be selected in such a way where it provides highest efficiency at the rated power.

Finally, FE analysis is performed for the previous and the optimally designed induction heating cookers to check characteristic performances. Various FEA results are compared and the effectiveness and accuracy of the proposed analysis and design method are verified.

\section{Proposed Idea}

Induction heating cooker heats the vessel by means of magnetic induction instead of thermal conduction from fire. The inductive heating has the capability to heat the vessel directly in a rapid way. Induction heating cooker uses input voltage $V_{\text {in }}$ with the frequency $f$ supplied from the drive circuit. The alternating magnetic field induces a current flow in the secondary winding (vessel) which heats the vessel. The practical induction heating cooker is shown in Fig. 1. In this study, the circuit parameters such as inductances and resistances can be calculated from the geometries. From these parameters equivalent circuit of induction heating cooker can be constructed easily to check the performance. 


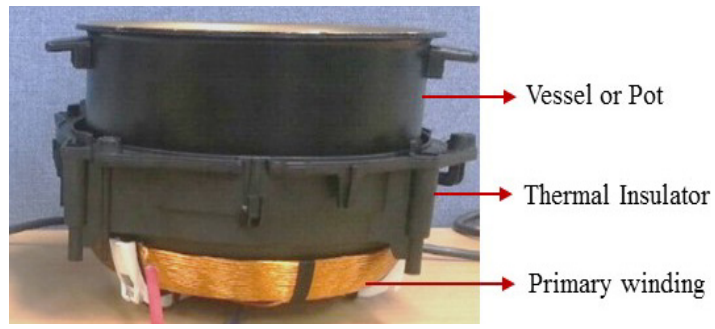

Fig. 1. Induction heating cooker model

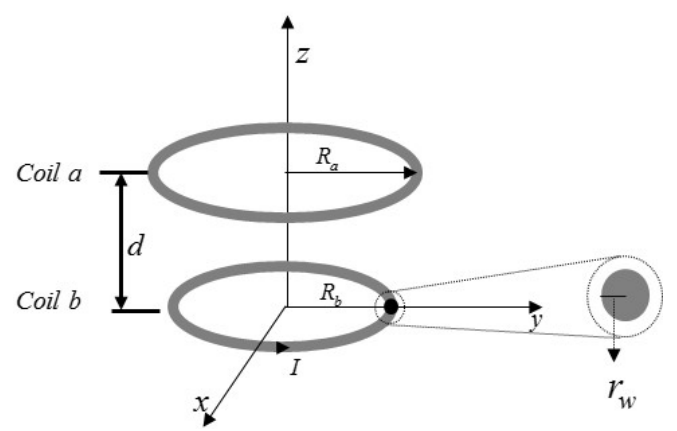

Fig. 2. Circular loop of wires

\subsection{Calculation of inductances}

In order to construct the equivalent circuit of induction heating cooker, it is necessary to calculate the resistances and inductances of the primary and secondary windings. For this purpose, the coaxial circular loop of wires is shown in Fig. 2.

The Biot-Savart law relates the magnetic fields to the currents which are their sources and it helps to calculate inductances for the given geometries of Fig. 2. But the Biot-Savart law's equation requires a dense sampling to solve it which consumes a lot of time. By using the standard numerical integration techniques, the simple analytical equation for the self-inductance can be obtained which provide the same results as the results of the equation obtained from Biot-Savart law, in a very short time [7]. The analytical equation for the self-inductance for the circular loop of wire is given as in (1).

$$
L_{a a}=\mu_{0} R_{a}\left[\ln \left(\frac{8 R_{a}}{r_{w}}\right)-2\right]
$$

where $r_{w}$ is the radius of wire, and $R_{a}$ is the radius of circular loop of coil $a$.

Similarly, the mutual inductance of magnetically coupled circular loops of wires as shown in Fig. 2 can also be calculated by using the simplified form of equation of mutual inductance (2), where $K$ and $E$ are complete elliptic functions [8].

$$
L_{a b}=2 \mu_{0} \frac{\sqrt{a+b}}{b}\left[\left(1-\frac{\beta^{2}}{2}\right) K\left(\beta^{2}\right)-E\left(\beta^{2}\right)\right]
$$

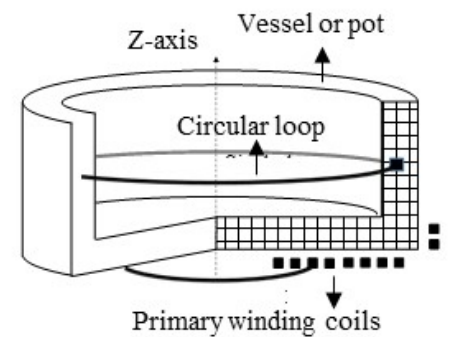

Fig. 3. Cut view of induction heating cooker

where $a=\frac{R_{a}^{2}+R_{b}^{2}+d^{2}}{R_{a}^{2} R_{b}^{2}}, \quad b=\frac{2}{R_{a} R_{b}} \quad$ and $\beta=\sqrt{\frac{2 b}{a+b}}$

where $R_{a}$ is the radius of one coil and $R_{b}$ is the radius of other coil $b$, and $d$ is the separation between these two coils.

\subsection{Construction of an equivalent circuit}

The equivalent circuit of induction heater is constructed with the resistances and inductances for the primary and secondary windings. To reduce the skin and proximity effect, primary winding coil is made of litz wire. The resistance of the primary winding can be calculated from its geometries by using common resistance law. The self and mutual inductances for the primary winding are calculated by using (1) and (2).

However, the secondary winding is the lump of solid conductor without any electric terminal, so it is impossible to use the previously established equations for the calculations of parameters. To obtained the parameters of the secondary winding, it is assumed that the secondary winding (vessel) is made of small pieces of slices which forms the circular loops of wire as shown in Fig 3. The depth of these circular loops of wires, is considered less than the skin depth to avoid the skin and proximity effects for the applied frequency. Consequently, the inductance equations (1) and (2) and the resistance law are applicable for each loop of wire to get the parameters of secondary windings.

\subsubsection{Voltage Equations of induction heating cooker}

For the given geometries of both primary and secondary windings, the resistance and inductance of each loop can be calculated. The voltage equation of $n^{\text {th }}$ loop of wires is expressed in phasor form, as in (3).

$$
\mathbf{V}_{n}=r_{n} \mathbf{i}_{n}+j \omega \sum_{k=1}^{N_{t o t}} L_{n k} \mathbf{i}_{k}\left(n \leq N_{t o t}\right)
$$

where $N_{t o t}$ is the sum of number of turns and $L_{n k}$ is the mutual inductance between the $n^{\text {th }}$ and $k^{\text {th }}$ conducting loops.

For convenience, the numbers from 1 to $N_{P}$ are assigned for the primary coils and the number from $N_{P}+1$ to $N_{\text {tot }}$ are assigned for the elements of secondary winding. The 
primary winding coils are connected in the series, so the currents of these circular loop of wires are same as $\mathbf{I}_{\mathbf{P}}$. The voltage equations for the coils of primary winding is given as in (4).

$$
\mathbf{V}_{i}=r_{i} \mathbf{I}_{P}+\left(j \omega \sum_{k=1}^{N_{P}} L_{i k}\right) \mathbf{I}_{P}+j \omega \sum_{k=N_{P}+1}^{N_{\text {tot }}} L_{i k} \mathbf{i}_{k} \quad\left(i \leq N_{P}\right)
$$

The voltage applied to the primary winding is the sum of all coils voltages $\mathbf{V}_{\mathrm{i}}$ because the coils of the primary winding are connected in series, which is given by (5).

$$
\begin{aligned}
\mathbf{V}_{P} & =\sum_{i=1}^{N_{P}} \mathbf{v}_{i} \\
& =\sum_{i=1}^{N_{P}}\left(r_{i}+j \omega \sum_{k=1}^{N_{P}} L_{i k}\right) \mathbf{I}_{P}+j \omega \sum_{k=N_{P}+1}^{N_{\text {tot }}}\left(\sum_{i=1}^{N_{P}} L_{i k}\right) \mathbf{i}_{k} \\
& =\mathbf{Z}_{P P} \mathbf{I}_{P}+\mathbf{Z}_{P S} \mathbf{I}_{S}
\end{aligned}
$$

where the secondary winding has the circular loop of wire in parallel so the current of coils of secondary winding is $\mathbf{I}_{S}=\left[\begin{array}{llll}\mathbf{i}_{N_{P}+1} & \mathbf{i}_{N_{P}+2} & . . & \mathbf{i}_{N_{\text {tot }}}\end{array}\right]^{T}$ and $\mathbf{Z}_{\mathrm{PS}}$ is the mutual impedance matrix between primary and secondary windings. $\mathbf{Z}_{\mathrm{PP}}$ is the impedance matrix of primary winding and it is given as in (6).

$$
\mathbf{Z}_{P P}=R_{P}+j X_{P}=\sum_{i=1}^{N_{P}} r_{i}+j \omega \sum_{i=1}^{N_{P}} \sum_{k=1}^{N_{P}} L_{i k}
$$

On the other hand, the secondary coils have the same voltage $\mathbf{V}_{\mathrm{s}}$ which is actually zero. The voltage equation for the elements of secondary winding is given as in (7).

$$
\begin{aligned}
\mathbf{V}_{S} & =v_{j}\left(N_{P}+1<j \leq N_{t o t}\right) \\
& =r_{j} \mathbf{i}_{j}+j \omega \sum_{k=1}^{N_{P}} L_{j k} \mathbf{I}_{P}+j \omega \sum_{k=N_{P}+1}^{N_{t o t}} L_{j k} \mathbf{i}_{k}
\end{aligned}
$$

The combined equation in the form of impedance matrices for the elements of secondary winding is given as in (8), [9].

$$
\mathbf{V}_{S}=\mathbf{Z}_{S P}^{T} \mathbf{I}_{P}+\mathbf{Z}_{S S} \mathbf{I}_{S}
$$

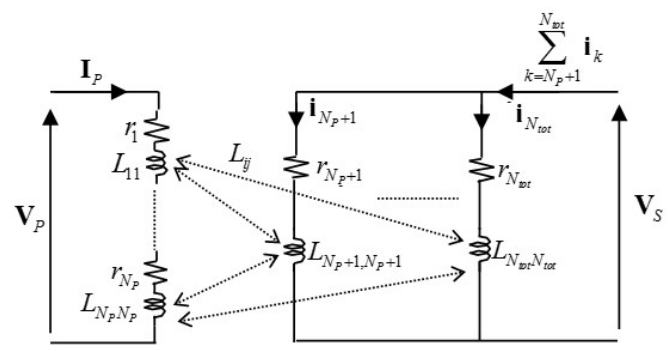

Fig. 4. Equivalent circuit of induction cooker for $N_{t o t}$ turns where $\mathbf{V}_{S}=\left[\begin{array}{llll}\mathbf{v}_{N_{p}+1} & \mathbf{v}_{N_{P}+2} & \ldots & \mathbf{v}_{N_{\text {tot }}}\end{array}\right]^{T}$.

Consequently, the equivalent circuit of induction heating cooker can be constructed with the derived equations (5) (8) as shown in Fig. 4.

By combining the (5) and (8) and with $\mathbf{V}_{\mathrm{S}}=0$, one can be obtained as in (9).

$$
\begin{aligned}
\mathbf{V}_{P} & =\left(-\mathbf{Z}_{P S}^{T} \mathbf{Z}_{S}^{-1} \mathbf{Z}_{P S}+\mathbf{Z}_{P P}\right) \mathbf{I}_{P} \\
& =\mathbf{Z}_{E Q} \mathbf{I}_{P}
\end{aligned}
$$

where $\mathbf{Z}_{\mathrm{EQ}}$ is the total impedance which can be seen from the primary side. The secondary impedance transferred to the primary side is also identified as in (10).

$$
\begin{aligned}
\mathbf{Z}_{E Q} & =\underbrace{\operatorname{Re}\left(-\mathbf{Z}_{P S}^{\mathbf{T}} \mathbf{Z}_{S}^{-1} \mathbf{Z}_{P S}+\mathbf{Z}_{P P}\right)}_{R_{E Q}}+j \underbrace{\operatorname{Im}\left(-\mathbf{Z}_{P S}^{\mathbf{T}} \mathbf{Z}_{S}^{-1} \mathbf{Z}_{P S}+\mathbf{Z}_{P P}\right)}_{X_{E Q}} \\
& =\underbrace{\operatorname{Re}\left(-\mathbf{Z}_{P S}^{\mathbf{T}} \mathbf{Z}_{S}^{-1} \mathbf{Z}_{P S}\right)}_{R_{S}}+R_{P}+j \underbrace{\operatorname{Im}\left(-\mathbf{Z}_{P S}^{\mathbf{T}} \mathbf{Z}_{S}^{-1} \mathbf{Z}_{P S}\right)}_{X_{S}}+j X_{P}
\end{aligned}
$$

where $R_{E Q}$ and $X_{E Q}$ are the equivalent resistance and reactance of induction cooker which can be seen from the primary side and $R_{S}$ and $X_{S}$ are the resistance and inductance of the secondary winding referred to the primary side.

Thus the final simplified equivalent circuit of induction heating cooker with secondary transferred to the primary side can be depicted as shown in Fig .5.

\subsubsection{Efficiency of induction heating cooker}

In order to get the efficiency of the induction heating cooker, it is necessary to calculate the total input and output powers of the induction heating cooker. By using the constructed equivalent circuit, its characteristic analysis is very easy to check the performance. The input power of induction heating cooker can be obtained by (11).

$$
P_{i n}=\operatorname{Re}\left(\mathbf{V}_{P} \mathbf{I}_{P}^{*}\right)=\operatorname{Re}\left(\mathbf{Z}_{E Q} \mathbf{I}_{P} \mathbf{I}_{P}^{*}\right)
$$

where $\mathbf{V}_{\mathrm{P}}$ is input supply voltage and $\mathbf{I}_{\mathrm{P}}{ }_{\mathrm{P}}$ is the conjugate of primary winding current $\mathbf{I}_{\mathrm{P}}$ which can be obtained from (9). The power delivered to the secondary winding can be calculated as in (12).

$$
P_{S}=P_{\text {in }}-P_{\text {loss }}=\operatorname{Re}\left(\mathbf{V}_{P} \mathbf{I}_{P}^{*}\right)-R_{P} I_{P}^{2}=R_{S} I_{P}^{2}
$$

Fig. 5. Simplified equivalent circuit of cooker 
The efficiency is the ratio between input and output powers of the induction heating cooker and it is given as in (13).

$$
\eta=\frac{P_{\text {out }}}{P_{\text {in }}}=\frac{R_{S} I_{P}^{2}}{\operatorname{Re}\left(\mathbf{V}_{P} \mathbf{I}_{P}^{*}\right)}=\frac{R_{S} I_{P}^{2}}{\operatorname{Re}\left(\mathbf{Z}_{E Q} \mathbf{I}_{P} \mathbf{I}_{P}^{*}\right)}=\frac{R_{S}}{R_{E Q}}
$$

All the above discussion described the simple method of analysis of induction heating cooker for the construction of equivalent circuit which is useful to find the performance of induction heating cooker in a very easy way.

\section{Optimization of Primary Winding Configuration}

The dimensions of the practical previous model which is to be optimized are shown in Fig. 7 and its specifications in detail are given in Table 1. In this study, with the given secondary (vessel) and the litz wire with given diameter, the primary winding is optimized to determine the best number of turns and their best positions. Due to the thermal insulator construction, maximum 22 conductors can be inserted at $R A$ area beneath the vessel and maximally 19 conductors can be inserted at $R B$ area to the side of the vessel as shown in Fig. 6, so maximum 41 turns in total can be inserted on the primary side for this model. An additional constraint for design is that all conductors in an area are contiguous together for manufacturing convenience.

Under these constraints, the number of total possible combinations $N_{\text {C.tot }}$ of primary winding coils for the available area is given by (14).

$$
\begin{aligned}
N_{C . t o t} & =\sum_{i=1}^{N_{a, \text { max }}}{ }_{\left(N_{a, \text { max }}-i+1\right)} C_{1} \times \sum_{j=1}^{N_{b, \text { max }}}\left(N_{b \text { max }}-j+1\right) \\
& =57684
\end{aligned}
$$

where $C$ denotes combination and $N_{a \text {.max }}$ and $N_{b . \max }$ mean the maximal number of conductors in $R A$ and $R B$ area respectively, that is, 22 and 19 . From the results of (14), it apparent that it is almost impossible to analyze all combinations with the FEM. However, with the proposed method the characteristics of induction cooker for each

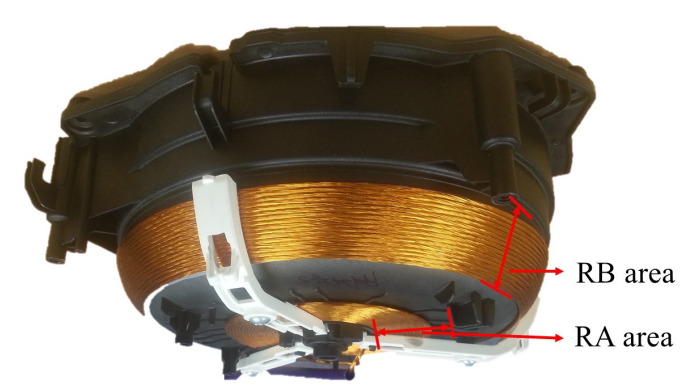

Fig. 6. Primary winding and thermal insulator structure combination can be obtained easily much faster than FE simulations.

The flow chart for the optimal designed of the primary winding coils is shown in Fig. 8. It is supposed that $N_{a}$ are the number of turns inserted in area $R A$ and $N b$ are the number of turns inserted at $R B$ area of the primary side of cooker. This flow chart shows that at the start secondary winding parameters such as resistance and inductances can be calculated from the given geometries. The minimum number of turns $\left(N_{a}=2, N_{b}=2\right)$ and their positions can be selected on $R A$ and $R B$ areas of the primary side. With the specific geometry of primary winding coils the secondary winding power and efficiency can be calculated. In this way the different number of combinations of primary winding can be obtained. The power and efficiency for each combination can be calculated. From those calculations the combination which has highest efficiency at rated power is selected as an optimized model which provide the number turns and their positions. The rated power for the design was selected as $1300 \mathrm{~W}$. In this paper the power for optimization is selected as in a very narrow range of $P_{\text {out }} \leq P_{\text {rated }} \pm 2.5 \%$ of $P_{\text {rated }}$. Among the combinations satisfying this range of power, the combination with the highest efficiency is selected. This is the simple analytical method which can be performed by using matlab program for simulation.

Fig. 9 and 10 show the power and efficiency graphs

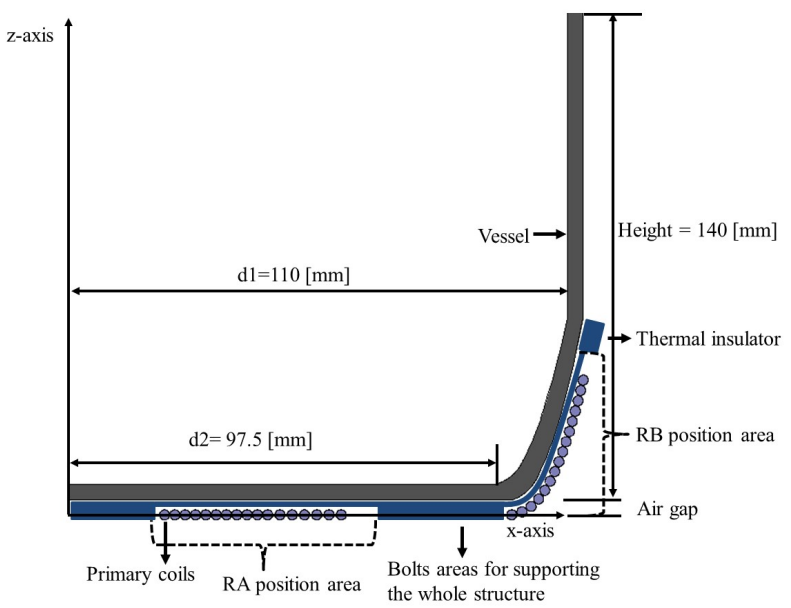

Fig. 7. Induction heating cooker with dimensions

Table 1. Specifications of analysis model

\begin{tabular}{c|c|c}
\hline \multicolumn{2}{c|}{ Input supply Voltage $V_{i n}$} & $177 \mathrm{~V}_{\mathrm{rms}}$ \\
\hline \multicolumn{2}{c}{ Input supply frequency $f$} & $20 \mathrm{kHz}$ \\
\hline \multicolumn{2}{c}{ Gap between primary and secondary } & $3.2 \mathrm{~mm}$ \\
\hline \multirow{4}{*}{ Primary winding } & Material & Copper \\
\cline { 2 - 3 } & Diameter of litz wire & $2.2 \mathrm{~mm}$ \\
\cline { 2 - 3 } & No. of strands & 23 \\
\cline { 2 - 3 } & $N_{a}$ & 17 turns \\
\cline { 2 - 3 } Secondary vessel & $N_{b}$ & 16 turns \\
\cline { 2 - 3 } & Material & Aluminum \\
\cline { 2 - 3 } & Thickness of vessel & $3.5 \mathrm{~mm}$ \\
\hline
\end{tabular}




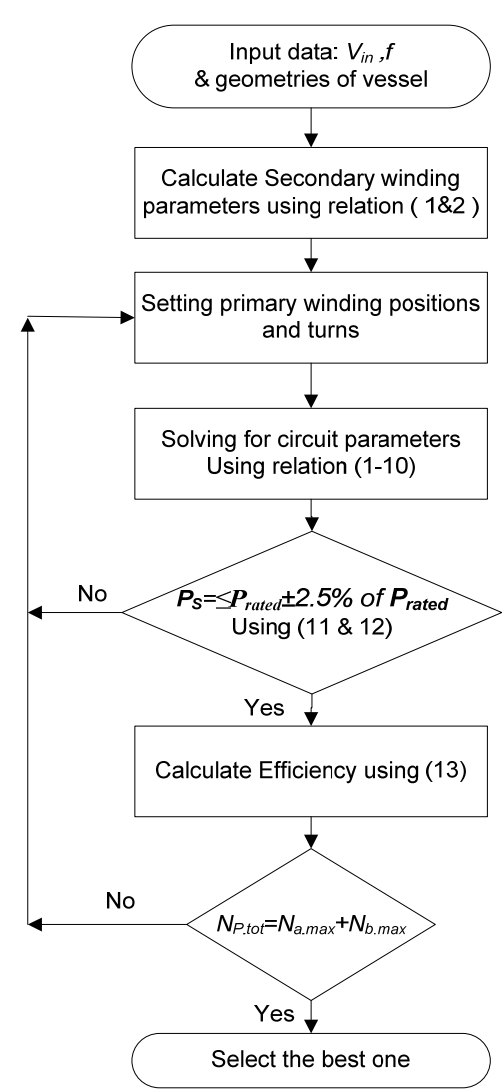

Fig. 8. Flow chart for optimization

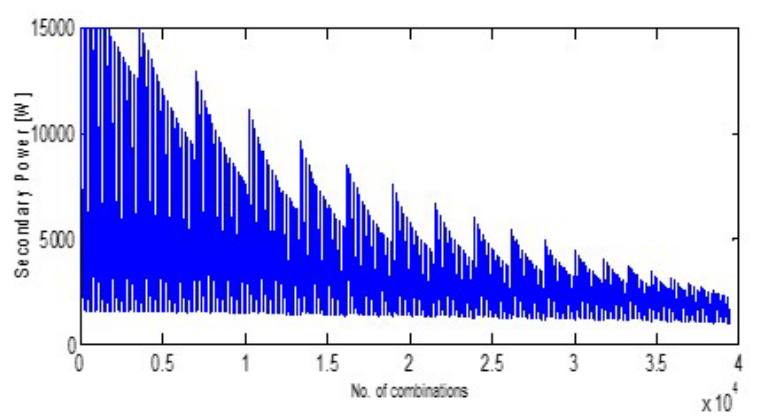

Fig. 9. Power vs no. of combinations

along the number of combinations of primary winding coils. The number of combinations is shown in these figures are less than the total number of combinations of (14) because the optimization is started from minimum 2 conductors $(N a=2, N b=2)$ for both RA and RB areas. The power and efficiency is varying along different combinations but there were only 416 combinations which satisfy the selected range of rated power.

The rated power was $1300 \mathrm{~W}$ but the power of optimized combination for the proposed design which has highest efficiency is $1329 \mathrm{~W}$. The proposed design and optimized method provide the information about the number of turns and their positions inserted at the primary side.

The total number of turns of primary winding for the optimized design is 30 , where $N_{a}=11$ and $N_{b}=19$ turns are

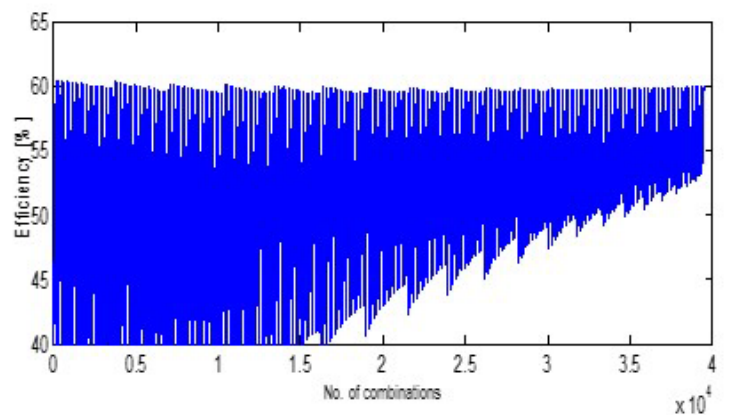

Fig. 10. Efficiency vs no. of combinations

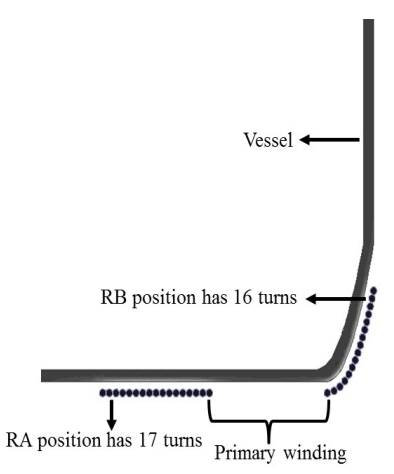

(a) Practical model

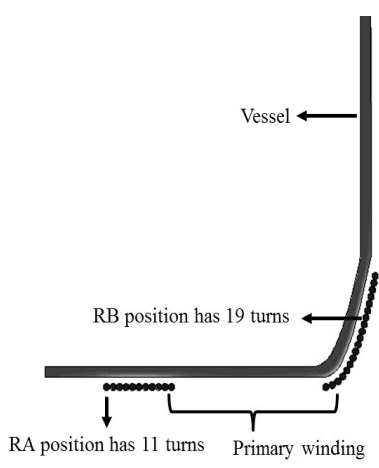

(b) Optimized model
Fig. 11. FEM models of induction heating cooker

inserted at the positions $R A$ and $R B$ respectively. The practical and optimized model are shown in Fig. 11.

\section{Verification Through FE-analysis}

The FE analysis is done with the RMS value of input supply $177 \mathrm{~V}$ at frequency of $20 \mathrm{kHz}$ for the optimized model which has the highest efficiency at rated power. The solution type of FE analysis is 2D time-transient with cylindrical coordination. The step size for the solution is kept very small as $(1 / 100 * 1 / 20000)$ sec. The Fig. 12 shows the flux lines, flux and current density distribution in the vessel and winding by using FE analysis. These figures clearly shows the magnetic induction current in the secondary conductor.

The input voltage, currents of primary and secondary winding are shown in Fig. 13 and 14 respectively. The input power is calculated from the waveforms of input voltage and current obtained from $\mathrm{FE}$ analysis as shown in Fig. 13. The RMS values of currents and voltage are also obtained from their waveforms. Apparent power is the multiplication of these RMS values of voltage and current. The input power can be extracted by taking the average of the point to point multiplication of current and voltage of the wave forms. The equivalent impedance from these waveform can be obtained as in (15) 


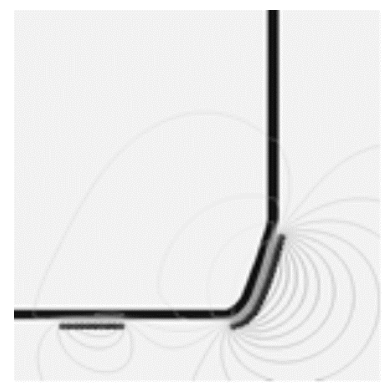

(a) flux density and lines

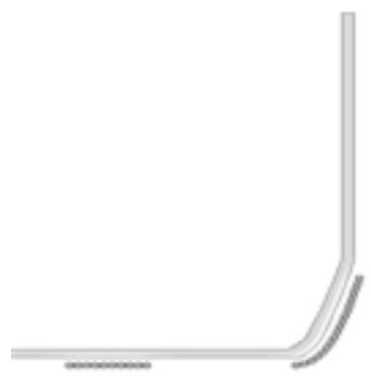

(b) current density
Fig. 12. Flux density and flux lines analysis

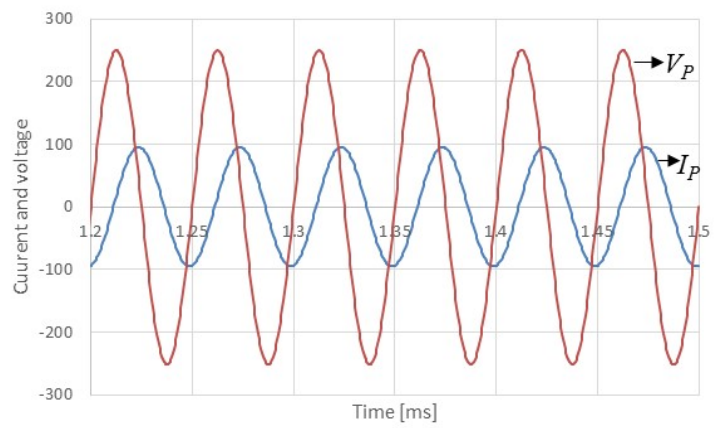

Fig. 13. Input voltage and current of primary winding

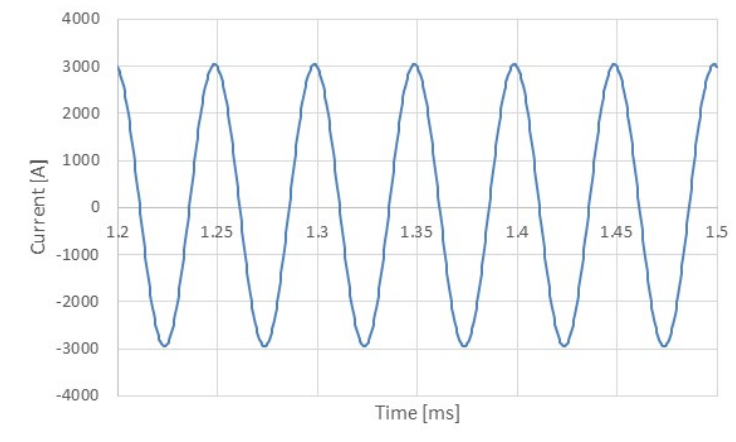

Fig. 14. Secondary winding current

$$
Z_{e q}=\frac{P_{a p p r a n t}}{I_{r m s}^{2}}(\cos \theta+j \sin \theta)
$$

where $\theta=\cos ^{-1}\left(\frac{P_{r e a l}}{P_{a p p r}}\right), \theta$ is the impedance phase angle,

$P_{\text {real }}$ is the real power and $P_{a p p r}$ is the apparent power. The secondary power can be calculated by subtracting the primary winding copper loss from input power.

The same FE analysis and characteristic analysis were carried out for the previous model, and the comparison of the analysis results for the both previous and optimized models, are given in the Table 2 and 3 respectively. From the results, it is shown that the error between analytical and FE analysis results is less than 5\% which might be caused by time step approximation and mesh qualities of $\mathrm{FE}$
Table 2. Analysis results of practical model

\begin{tabular}{c|c|c}
\hline Parameters & Analytical results & FEM Results \\
\hline$I_{P}$ & $69.3 \mathrm{~A}$ & $68.9 \mathrm{~A}$ \\
\hline$I_{S}$ & $2103 \mathrm{~A}$ & $2083 \mathrm{~A}$ \\
\hline$P_{S}$ & $1300 \mathrm{~W}$ & $1321 \mathrm{~W}$ \\
\hline efficiency & $66.1 \%$ & $66.3 \%$ \\
\hline
\end{tabular}

Table 3. Analysis results of the optimized model

\begin{tabular}{c|c|c}
\hline Parameters & Analytical results & FEM Results \\
\hline$I_{P}$ & $68.6 \mathrm{~A}$ & $67.4 \mathrm{~A}$ \\
\hline$I_{S}$ & $2084 \mathrm{~A}$ & $2067 \mathrm{~A}$ \\
\hline$P_{S}$ & $1329 \mathrm{~W}$ & $1341 \mathrm{~W}$ \\
\hline efficiency & $67.0 \%$ & $67.5 \%$ \\
\hline
\end{tabular}

analysis. The efficiency of optimized model is about $1 \%$ higher and number of turns are decreased to 30 from 33, which shows the validity of proposed idea.

\section{Conclusion}

In this paper, the novel method of analysis and optimal design of induction cooker is investigated in detail which is useful and time saving. The proposed method is started from the equivalent circuit analysis to find the electrical parameters and then the primary winding coils are designed with the simple optimized method.

Finally, FE analysis is performed for the best optimized combination of the primary winding coils. From the results it can be concluded that rated power satisfy with the analytically calculated one as well as the number of primary winding and their position of insertion is also obtained to get the maximum efficiency at rated power. The proposed method can be implemented by the designers easily for the optimal design of induction heating cooker of different ratings.

\section{Acknowledgements}

This work was supported by the Energy Efficiency \& Resources Core Technology Program of the Korea Institute of Energy Technology Evaluation and Planning (KETEP), granted financial resource from the Ministry of Trade, Industry \& Energy, Republic of Korea (No. 201420204060).

\section{References}

[1] Lichan Meng, Ka Wai Eric Cheng, Ka Wing Chan, "Systematic Approach to High-Power and EnergyEfficient Inductrial Induction Cooker System: Circuit Design, Control strategy, and Prototype Evaluation." IEEE Transection on power Electronics, Vol.26, No. 
12, December 2011.

[2] L.C. Meng, K.W.E Checng and K.W. Chan, "Heating Performance Improvement and field Study of Induction Cooker"3rd international conference on power Electronics System and Applications 2009.

[3] Bekir Sami Sazak and Sevilary, "Reducing the Number of Measurements in induction cooker Design" ICEMI'2009.

[4] Jin-kyu Byun, Kyung Choi, Hee-Succ Roh, and Song-yop Hahn "Optimal Design Procedure for a Practical Induction Heating Cooker", IEEE Transactions on Magnets, vol, 36, No.4,July 2000.

[5] Dong-Seong Kim, Ji-Young So, and Dae-kyong Kim "Study on Heating Performance Improvement of Practical Induction Heating Rice Cooker with Magnetic Flux Concentrator", IEEE Transactions on applied superconductivity, Vol. 26, No, June 2016.

[6] Rachard Yi Zhang. "A Generalized Approach to Planner Induction Heating Magnet”, Massachusetts Institute of technology June 2012.

[7] Nobbey Stevens, Lieven Destrycker and Werner Verschelde, "Procedure to Calculate the Inductance of a Circular Loop near Metal Plate”, ISSN 2011.

[8] Kurt Nalty, "Calassical Calculation of Mutual Inductance of two Coaxial Loop in MKS Units", Austin, TX 78750 USA, March, 6, 2011.

[9] Guo-Quan Zhou, "The Equivalent Self Inductance of N coupled Parallel coils", Progress in Electromagnetic Research Letters 2014.

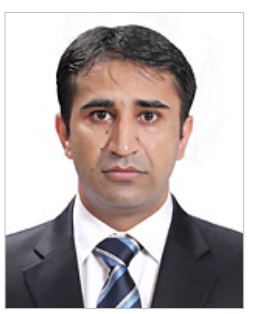

Muhammad Humza $\mathrm{He}$ was born in Jampur (Punjab), Islamic Republic of Pakistan. He received his Bachelor's degree in electrical engineering from FUUAST Islamabad, Islamic Republic of Pakistan in 2012. Now he is working towards his Ph.D. degree in electrical engineering at Kunsan National University. His research interests includes electric machines, and power electronic.

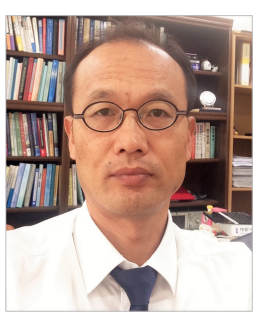

Byungtaek Kim He was born in Seoul, Korea. He received the Bachelor's, Master's, and Ph.D. degrees in electrical engineering from Hanyang University, Seoul, Korea, in 1994, 1996, and 2001, respectively. He was an Electrical Engineer with the R\&D center, Samsung Electro-Mechanics Company, Ltd., and with the DA research center, LG Electronics Inc., for ten years. Since 2005, he has been a Professor of electrical engineering with the Department of Electrical Engineering, College of Engineering, Kunsan National University, Kunsan, Korea. In 2012, he was a Visiting Scholar with the Department of Electrical and Computer Engineering, College of Engineering, University of Wisconsin-Madison, Madison, WI, USA. His research interests include electric machines and power electronics. 